Documentation et bibliothèques

\title{
Colloque d'orientation des bibliothèques publiques : Sainte-Foy les 11 et 12 septembre 1997
}

\section{Réjean Savard}

Volume 44, numéro 2, avril-juin 1998

URI : https://id.erudit.org/iderudit/1032960ar

DOI : https://doi.org/10.7202/1032960ar

Aller au sommaire du numéro

Éditeur(s)

Association pour l'avancement des sciences et des techniques de la

documentation (ASTED)

ISSN

0315-2340 (imprimé)

2291-8949 (numérique)

Découvrir la revue

Citer cet article

Savard, R. (1998). Colloque d'orientation des bibliothèques publiques :

Sainte-Foy les 11 et 12 septembre 1997. Documentation et bibliothèques, 44(2),

93-96. https://doi.org/10.7202/1032960ar

Tous droits réservés (C) Association pour l'avancement des sciences et des techniques de la documentation (ASTED), 1998
Ce document est protégé par la loi sur le droit d'auteur. L'utilisation des services d'Érudit (y compris la reproduction) est assujettie à sa politique d'utilisation que vous pouvez consulter en ligne.

https://apropos.erudit.org/fr/usagers/politique-dutilisation/ 


\title{
Colloque d'orientation des bibliothèques publiques Sainte-Foy les 11 et 12 septembre 1997
}

\author{
Réjean Savard \\ École de bibliothéconomie et des sciences de l'information (EBSI), Université de Montréal
}

\section{Les préparatifs}

Depuis quelques années déjà circulait l'idée de tenir des "états généraux" sur la situation des bibliothèques publiques au Québec. D'abord, l'Association des bibliothèques publiques avait envisagé d'organiser un tel événement, mais avait dû abandonner faute de ressources disponibles; puis, les Centres régionaux de services aux bibliothèques publiques (CRSBP) avaient également proposé de tenir un colloque d'orientation. C'est d'ailleurs à la suite d'une rencontre avec leurs représentants et la ministre de la Culture et des Communications, Louise Beaudoin, au printemps 1996 que le projet fut entériné.

Afin de garantir la plus grande ouverture possible à tous les milieux concernés par la lecture publique, le Ministère, qui avait pris en charge le projet, constitua un comité d'orientation formé de représentants des principaux regroupements, associations et intervenants reliés au milieu des bibliothèques publiques. Présidé par Denis Delangie, responsable du secteur des bibliothèques au Ministère, ce comité avait pour mandat de départ de "prendre toutes les mesures nécessaires pour assurer la réussite du colloque et de façon plus spécifique:

- de préciser les objectifs poursuivis avec la tenue du colloque;

- d'identifier les thèmes qui favoriseront des discussions fructueuses;

- d'assurer la production des documents de support et, au besoin, faire appel à des groupes de travail pour approfondir des thèmes de discussion;

- d'élaborer le programme du colloque;

- de déterminer les participants du colloque et d'effectuer les invitations."

Les travaux du Comité commencèrent à l'automne 1996, avec pour objectif la tenue du Colloque au printemps 1997.
Les premières réunions permirent à tous les membres de s'entendre sur les objectifs de l'événement. Cinq grands thèmes de discussion furent adoptés, et pour chacun de ceux-ci, cinq sous-comités furent chargés d'en explorer les enjeux et de préparer les documents pour les futurs participants au Colloque.

Mais en janvier 1997, il apparaissait à certains membres du Comité que cette façon de travailler ne permettrait pas de rencontrer l'échéancier prévu. La date du Colloque fut reportée de quelques mois et la méthode de travail par sous-comités, abolie. Les travaux se poursuivirent jusqu'au printemps et c'est le Ministère avec la collaboration de l'ASTED qui finalisa l'organisation du Colloque pour les 11 et 12 septembre suivants.

\section{Le Colloque}

Le matin du 11 septembre, plus de 200 personnes étaient inscrites, dont environ une trentaine d'élus municipaux, ce qui constituait un succès en soi. Une certaine fébrilité régnait chez les participants. L'animatrice recrutée par le Comité d'orientation brisa la glace en souhaitant la bienvenue à tous et en donnant les consignes générales pour le fonctionnement. Vint ensuite l'allocution de la ministre Louise Beaudoin. À la grande satisfaction de toutes les personnes présentes, celle-ci réaffirma avec force la volonté de son gouvernement de ne pas abandonner la lecture publique et de maintenir son leadership en ce qui a trait aux bibliothèques publiques. Louise Beaudoin souligna l'importance qu'elle accordait à la Politique de la lecture actuellement en préparation dans le contexte d'une société où le savoir et, par conséquent, les habilités de lecture doivent être priorisés. Elle ajoutait que des «ajustements importants" devraient être faits dans le système actuel, évoquant notamment une révision éventuelle du mandat des CRSBP. Le Colloque était lancé.
Vint ensuite une table-ronde où trois personnes étaient chargées d'aiguillonner les débats à venir. Roger Carette, dynamique maire d'une municipalité de la région de Québec, présenta une intervention d'où émanait un certain questionnement quant à la responsabilité des bibliothèques publiques au Québec: il termina en soulignant que celle-ci débordait les juridictions locales ou les compétences municipales. Dans son intervention, France Bouthillier, professeure adjointe à l'École de bibliothéconomie de l'Université McGill s'interrogeait sur le rôle des bibliothèques publiques dans une société de l'information face aux nouvelles technologies. Enfin, l'auteure et journaliste Dominique Demers ramena le débat à la question fondamentale de la lecture en parlant du sujet de son dernier livre La mystérieuse bibliothécaire. Complétant son intervention avec des anecdotes personnelles tout aussi appropriées que l'histoire de son roman, elle affirma, au grand plaisir des participants, que la chose la plus importante en ces temps de restrictions budgétaires était certainement de «faire vivre la lecture".

Suivit une période de questions et d'échanges quelque peu influencée par la dernière intervention. Plusieurs intervenants réaffirmèrent que la mission de base de la bibliothèque devrait être recentrée autour de la lecture. L'importance de faire le lien entre le lecteur et le livre, notamment par la promotion, fut également mentionnée à plusieurs reprises. Quelqu'un posa la question suivante: "Exploite-t-on suffisamment les collections déjà disponibles?" L'importance de partager les ressources fut évoquée plusieurs fois. Somme toute, une entrée en matière diversifiée qui laissait entrevoir de riches échanges pour la suite du Colloque.

Une série de quatre thèmes à débattre en ateliers sous la responsabilité d'animateurs professionnels recrutés pour 
l'occasion, constituait l'essentiel de l'événement. Ces ateliers étaient offerts à deux reprises: en après-midi la première journée et une deuxième fois, l'avant-midi de la deuxième journée, de sorte que les participants avaient la possibilité de s'exprimer sur deux thèmes de leurs choix.

- ATELIER A: Le premier thème à débattre portait sur «Les usagers des bibliothèques et les services aux citoyens". Dans cet atelier, les participants semblaient assez d'accord pour dire que les bibliothèques répondent assez bien aux besoins des citoyens québécois. Ils pensent cependant que la fréquentation des bibliothèques pourrait être encore augmentée. Pour ce faire, quatre moyens principalement ont été discutés. D'abord, trouver des façons de mieux connaître les besoins des clientèles actuelles et potentielles en raffinant les outils déjà existant ou en développant des outils nationaux et locaux, incluant des méthodes d'évaluation. Deuxièmement, s'attaquer aux barrières qui nuisent à l'accès des bibliothèques comme la tarification ou encore le manque de ressources humaines qui limite le temps consacré aux clientèles. Troisièmement, mettre davantage en commun les expériences ayant permis d'accroître la clientèle. Enfin et surtout, mettre sur pied, avec les partenaires appropriés, une campagne de publicité et de relations publiques d'envergure qui permettrait de mieux faire connaître les services des bibliothèques et d'entraîner éventuellement une augmentation de la fréquentation.

- ATELIER B : L'atelier portait spécifiquement sur la coopération et la mise en commun des ressources. Sur ce point, les discussions ont été parfois laborieuses et les consensus assez rares, mais tous les participants ont reconnu l'importance de coopérer davantage et d'établir des réseaux plus fonctionnels. En ce qui concerne les obstacles à la coopération, plusieurs participants ont indiqué qu'il ne doit pas y avoir de modèle unique et que la coopération peut prendre des formes différentes selon, par exemple, la taille des municipalités. En ce qui concerne l'élargissement du rôle des CRSBP, les opinions vont d'un oui conditionnel à un non catégorique, alors que plusieurs participants déplorent l'absence de véritables structures organisationnelles pou- vant faciliter la coopération. Selon certains, il faudrait effectuer des études de faisabilité pour faire avancer l'idée de coopération au Québec et éventuellement modifier les mentalités.

Quant aux domaines de coopération mentionnés en priorité par les participants à ce deuxième atelier, on a parlé surtout d'informatisation; celle-ci se retrouvant comme objectifs prioritaires de coopération pour le traitement documentaire, le prêt entre bibliothèques et la rationalisation des acquisitions et des collections. Quant à la coopération scolaire-municipale, on a parlé de mise en commun de certaines ressources, de moyens pour faciliter l'accès aux livres pour les enfants, de partage de la technologie, d'activités d'animation, mais en général les participants ont semblé méfiants face à ce sujet, rejetant la fusion des bibliothèques scolaires et municipales.

On souhaite donc plus de coopération, mais par des mesures qui respectent les différentes parties prenantes, et on ajoute que ces mesures se doivent d'être incitatives et non coercitives. Enfin, certains se sont dits d'accord pour affirmer qu'il manque un «chef d'orchestre» pour orienter et mettre en place cette coopération entre bibliothèques de lecture publique au Québec. À cet égard, d'autres intervenants ont répliqué que c'est à l'État d'être la bougie d'allumage.

- ATELIER C: L'atelier portait sur les nouvelles technologies de l'information et des communications. En ce qui concerne ce thème, deux éléments principaux ont retenu l'attention des participants. II faut d'abord s'assurer que l'automatisation des bibliothèques est complétée et deuxièmement, s'assurer de la compatibilité des logiciels utilisés, notamment par l'adoption de normes internationales reconnues telle la norme Z39.50. Ainsi les bibliothèques du Québec pourront-elles garantir un meilleur accès aux catalogues des bibliothèques, automatiser les procédures de prêt entre bibliothèques, repêcher des notices pour le traitement des documents, etc. Selon plusieurs participants, l'État a encore là un rôle important à jouer. En ce qui a trait à Internet, plusieurs participants ont souligné l'importance pour la bibliothèque de s'impliquer, notamment en ce qui concerne la forma- tion des citoyens aux nouvelles technologies. On mentionne l'importance de la bibliothèque virtuelle pour l'avenir et l'obligation pour les bibliothèques publiques de participer à son développement en coopération avec les organismes nationaux comme la Grande Bibliothèque du Québec. La formation du personnel a aussi retenu l'attention des participants à cet atelier: étant donné son importance en cette période de grands changements, celle-ci pourrait être plus facilement accessible et faire appel à davantage de coopération entre tous les partenaires (universités, collèges, associations professionnelles; entreprises).

ATELIER D : L'atelier avait comme toile de fond le difficile contexte budgétaire des municipalités québécoises; le dernier atelier portait sur le financement des bibliothèques publiques. Dans cet atelier, on a d'abord discuté des responsabilités de l'État versus celles des municipalités. II n'apparaît pas réaliste ou souhaitable que l'État se retire du financement des bibliothèques publiques au seul profit des municipalités, son rôle de leader étant perçu comme crucial pour la majorité des participants. Celui-ci devrait exercer ce rôle, par exemple en élaborant les politiques et en établissant les normes. II devrait maintenir son financement au développement des collections et à la construction de bibliothèques, soutenir les initiatives de coopération et intervenir afin d'atténuer les disparités entre les régions et entre les municipalités. Les municipalités, quant à elles, devraient intervenir de manière à assurer le bon fonctionnement de la bibliothèque en vue de dispenser à la population locale des services de qualité.

Le statut des bibliothèques (municipal ou corporation autonome) a aussi fait l'objet de débats où se sont affrontés partisans et réticents, sans toutefois parvenir à un consensus.

L'ajout de nouveaux revenus était aussi à l'ordre du jour. Certains participants ont souligné l'importance de la tarification dans ce contexte, alors que d'autres ont fait remarquer la diminution des clientèles qu'entraînait cette dernière mesure et affirmé qu'elle représentait, par conséquent, un frein à l'accessibilité. D'autres ont prôné la recherche de nouvelles sources de financement, notam- 
ment le recours aux fondations et aux commandites.

\section{La plénière}

En après-midi de la deuxième journée, les participants retournèrent en plénière. Chaque animateur vint d'abord faire un compte rendu des ateliers. Ensuite, l'animatrice expliqua comment trois personnes avaient été nommées par le comité d'orientation afin d'élaborer une déclaration-synthèse permettant de conclure le colloque. Ces personnes avaient assisté aux différents ateliers, avaient recueilli les synthèses de chacun des rapporteurs assignés et avaient rédigé un texte qui avait été reproduit sur transparents pour être présenté en plénière aux participants par le responsable de l'animation du colloque. Ce texte est reproduit en encadré.

Les réactions des participants furent pour le moins ambiguës, chacun s'attendant à retrouver dans cette synthèse un certain nombre d'éléments précis. Certaines personnes manifestèrent leur insatisfaction, alors que d'autres - on peut estimer qu'il s'agit de la majorité - se rallièrent aux principes de cette déclaration.

\section{Conclusion}

Dans l'ensemble, on peut conclure qu'une réelle volonté de changement était perceptible chez les participants à ce Colloque. Plusieurs ont demandé qu'il y ait des suites aux échanges et aux propositions qui ont été faites à cette occasion. On a notamment proposé de mettre sur pied des comités de travail afin de pousser plus loin la réflexion et proposer des mesures concrètes. Le souhait général était que la politique de la lecture actuellement en préparation tienne compte des opinions exprimées lors de ce Colloque.

res 4 net une interface installée sur le Web du catalogue de la Bibliothèque nationale du Canada (BNC). L'interface resAnet fournit de courtes notices décrivant des documents de la collection de la Bibliothèque nationale.

Les caractéristiques de resAnet comprennent entre autres :

- des liens hypertextes à des ouvrages, des vedettesmatières et des documents connexes dans la collection de la Bibliothèque nationale;

- la capacité de faire le tri des résultats par titre, par auteur et par date ;

- la fonction de recherche par mots clés ;

- la capacité de limiter les recherches par date, par langue, par lieu de publication ou par type de document.

On peut y avoir accès gratuitement par l'entremise du Service Web de la BNC à :

\section{www.amicus.nlc-bnc.ca/resanet}




\section{Colloque d'orientation des bibliothèques publiques tenu à Québec, les 11 et 12 septembre 1997}

\section{Déclaration : un virage à réaliser}

Dans un contexte où le niveau d'éducation et de culture d'une société se mesure à la capacité de chaque citoyen de s'épanouir, de comprendre et de penser le développement de la société, cette capacité du citoyen exige l'utilisation et la maîtrise de la lecture.

De plus, parce que la mondialisation des marchés prend une ampleur sans précédent, que les emplois se transforment constamment et exigent des compétences de plus en plus précises, les citoyens sont amenés à développer leurs compétences et à utiliser les technologies de l'information. La lecture demeure donc l'outil essentiel du monde du travail.

\section{Considérant}

- les priorités actuelles du gouvernement du Québec: éducation, culture et développement de la main-d'œuvre et de l'emploi;

- la vision de $M^{\text {me }}$ Louise Beaudoin, ministre de la Culture et des Communications, exprimée lors du Colloque d'orientation des bibliothèques publiques le 11 septembre 1997, et la préparation d'une politique de la lecture;

- le Manifeste de l'UNESCO et la mission des bibliothèques publiques;

- les communications des panelistes, les témoignages et opinions présentés en plénière ainsi que les visions communes émergeant des discussions en ateliers.

Au terme de ce Colloque, nous énonçons que:

- la lecture, constituant un des principaux modes d'apprentissage et d'expression, doit être priorisée par l'État qui doit en faire la promotion auprès de tous les citoyens et en favoriser la pratique de concert avec les milieux locaux, éducationnel et municipal;

- la bibliothèque publique constitue la plaque tournante et l'outil privilégié d'une stratégie de développement de la lecture, tant pour des fins d'information, d'éducation, de formation continue, d'enrichissement culturel et de loisir.

- la bibliothèque publique relève d'une responsabilité collective assumée par l'État, la communauté locale et le citoyen.

Pour remplir son mandat, la bibliothèque publique doit donc poursuivre son développement en ajustant ses services, aux besoins des citoyens d'aujourd'hui et de demain ainsi qu'à l'environnement dans lequel ils évoluent. Pour ce faire, des modifications aux structures actuelles devront être apportées.
Au terme de ce Colloque regroupant l'ensemble des intervenants et des partenaires reliés au développement des bibliothèques publiques, les participants souhaitent que:

1. L'État québécois, appuyé sur les principes d'équité et d'accessibilité, assume le leadership du développement des bibliothèques publiques.

Pour ce faire, l'État devrait plus spécifiquement:

- assurer l'élaboration d'une politique nationale ainsi que des cadres de référence et des normes;

- soutenir le développement des collections;

- soutenir des initiatives de coopération entre les bibliothèques publiques et leurs partenaires;

- mettre en œuvre des programmes incitatifs pour aider les bibliothèques publiques à atteindre les normes nationales.

2. La municipalité assume la responsabilité d'une prestation de services de qualité

- dans une optique d'approche client;

- dans une optique de concertation et de partenariat avec les intervenants scolaires, socio-communautaires et privés constituant sa communauté locale.

3. Le citoyen participe activement à définir l'avenir de la bibliothèque publique au Québec et celui de sa bibliothèque locale en particulier en s'impliquant de plus en plus dans l'administration et la définition des services à offrir en devenant ainsi un acteur majeur de la promotion et du développement de sa bibliothèque locale et de l'ensemble des bibliothèques publiques.

4. La bibliothèque publique assume son rôle de responsable de la promotion et de la diffusion de la lecture :

- d'abord, en participant à une campagne nationale de promotion de la lecture et de l'ensemble des services;

- en initiant les jeunes au plaisir de lire;

- en développant la passion de la lecture par des activités appropriées, organisées en coopération avec la communauté locale.

La bibliothèque développe la coopération et le réseautage dans un objectif d'amélioration des services, de plus grande accessibilité et de partage des ressources:

- traitement documentaire

- PEB

- animation et promotion

- rationalisation des collections

La bibliothèque constitue un guichet unique quant à l'information communautaire;

La bibliothèque devient un lieu de formation pour les nouvelles technologies.

En conclusion, les participants au Colloque d'orientation des bibliothèques publiques du Québec ont clairement manifesté leur volonté de changement, de coopération et de réseautage. 